\title{
The future of medical imaging in the detection of early markers of disease
}

\author{
Barbara Y. Croft* \\ Cancer Imaging Program, National Cancer Institute, National Institutes of Health, Rockville, MD 20852, USA
}

\begin{abstract}
Imaging techniques are a combination of a contrast mechanism, exogenous or endogenous, and an instrument to exploit that contrast. This final chapter of these two special issues of this journal points to possible ways to improve the ability of imaging systems to exploit markers of cancer in the early detection of that disease. The aim not only is to find cancer at an earlier, more treatable stage, but to determine whether the disease discovered is dangerous and to indicate the possibilities for successful treatment. These topics are explored for each imaging system, with an emphasis on directions for future improvements.
\end{abstract}

\section{Introduction}

As these two special issues of Disease Markers on the Functional Imaging of Early Markers of Disease illustrate, the ability of imaging techniques to detect early disease using disease markers varies greatly. To sharpen the focus as we look to the future, consider the disease progression to be imaged and how that might be done. Using cancer as a model of disease progression, consider Fig. 1. To be successful at very early detection, we must see lesions as they are transforming from a pre-cancerous state to a cancerous state; in actuality, detection at the fully formed but not spreading stage is acceptable. Imaging methods can enable detection before clinical symptoms appear by taking advantage of the contrast and sensitivity that the methods allow. Disease markers can enable specificity, contrast, and sensitivity. A key question is, can we combine increases in contrast and sensitivity without "opening the floodgates" to false positives in the future?

Imaging in the clinical and research arenas varies

*Address for correspondence: Barbara Y. Croft, Cancer Imaging Program, National Cancer Institute, National Institutes of Health, 6130 Executive Boulevard, EPN 6064, Bethesda, MD 20892-7412, USA, (FedEx: Rockville, MD 20852-4910). Tel.: +1 301496 9531; Fax: +1 301480 3507; E-mail: croftb@mail.nih.gov. greatly with the modality. This chapter will discuss, compare, and contrast what and how the imaging modalities currently are diagnosing and point to directions in which they might go. The obvious prediction for the future of imaging is that it will be faster with better resolution, but this is too facile and does not give credit to the possibilities of imagination and technological innovation. I will try to go beyond speed and small pixels into more fanciful areas, both as a suggestion for what to look for and as a suggestion for ways in which research might be directed.

The ability of the human eye and brain to immediately appreciate the results that come from these modalities varies widely. One can look at the x-ray of a broken bone, captured on film, and make a diagnosis. One cannot make an equivalent image with a laser light shining through or reflected back from that same area; a computer is required to analyze the data before an image can be appreciated. These differences do not mean that the directly viewed technique is normal or true and that the computer-mediated one is abnormal or "black magic." The same differences occur in astronomy between optical telescopes and other cameras that view the sky. Astronomers have learned to convert the pictures from the other instruments into images like those they are accustomed to viewing to help us appreciate the results. The same must be done with medical images. 


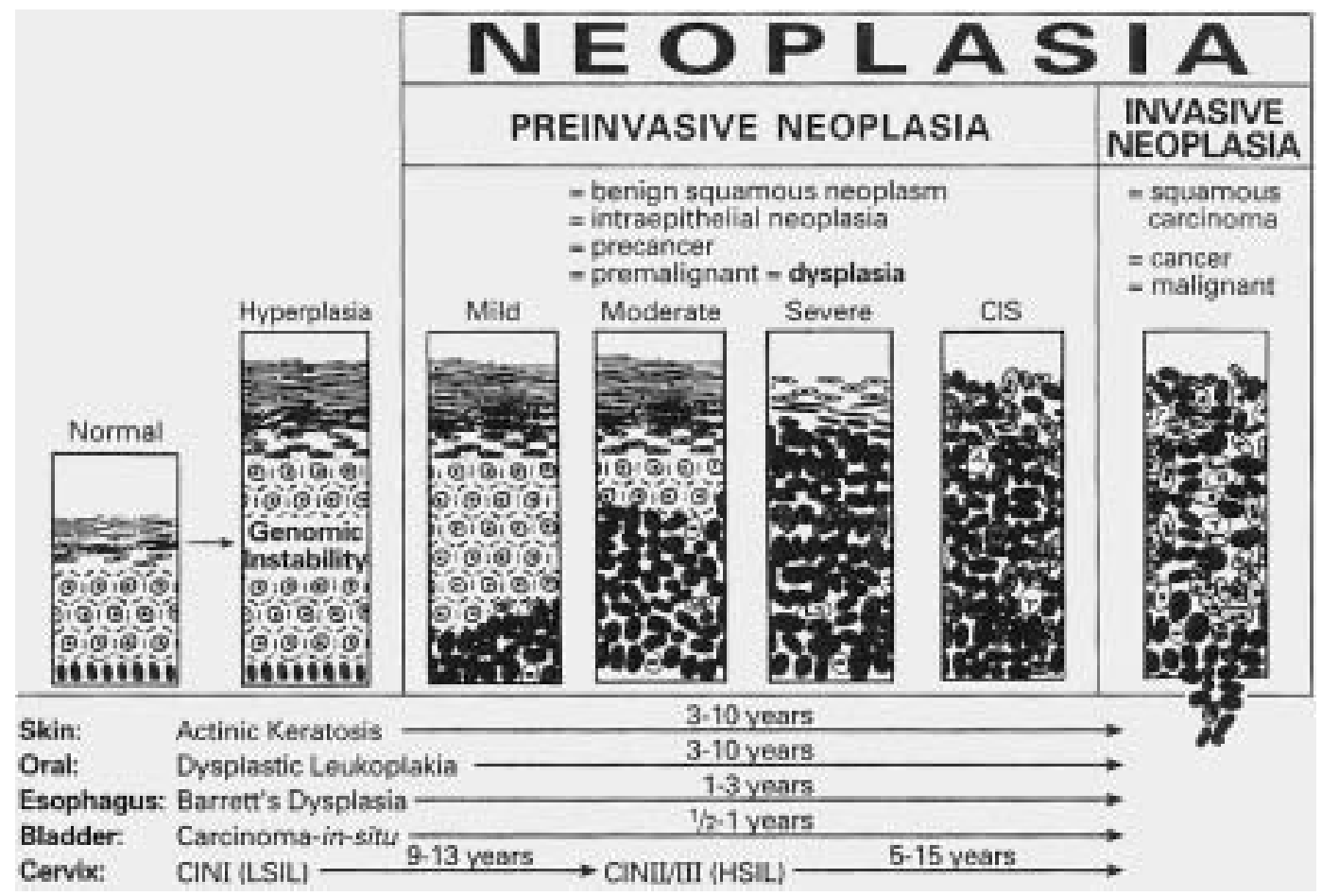

Fig. 1. Diagram of neoplasia developing in squamous epithelium (used with permission [34]).

\section{The development of cancer: Matching signal and sensor}

Look at the classic diagram of the development of cancer, Fig. 1. While there are other diagrams with different cell arrangements, the typical progression is from normal to hyperplasia, to stages of pre-invasive neoplasia ranging from mild through moderate to severe, and then to invasive neoplasia. Note the year markers and the indicated times. If $1 \mathrm{~cm}$ in size, 1 gram in weight, and 30 doubling times are considered typical of invasive neoplasia, and three to 20 years are considered typical times to achieve this size, then the doubling time is measured in months from one to eight. To find this tumor at $10^{+6}$ cells or 1 microgram in weight is 20 doubling times. This would mean finding lesions one to seven years before their clinical presentation. This "back-of-the-envelope" calculation assumes that the doubling time does not change with the progression of the tumor. To find tumors that are 1 microgram in size implies a large signal-to-noise ratio, but this may be achieved by having a large signal or a small noise. Larger signals can be achieved by signal amplification and enhancement. The best way to decrease noise is to use techniques that have inherently low noise, to cool the detectors, and to apply prior or other knowl- edge about the imaging situation to subtract the noise as background. Such a signal need not accurately define the size of the lesion but needs to locate the lesion and say something about the conversion to malignancy.

Cancer may be detected by biochemical means from blood or urine samples by measuring the quantity of a unique marker substance. This is the ideal situation, since the processing of such samples has become automated and routine, making it inexpensive for screening. The point of the research results discussed in this journal is to find these markers and exploit them. The point of the two special issues of which this contribution is a part is to discuss how imaging methods might aid this process.

Another long-standing screening method is the analysis of biopsy samples from body sites that are easy to reach, such as the mouth or uterine cervix. Detection of cervical cancer or pre-cancer is possible because of the accessibility of the cervix; the PAP smear is very important in cancer detection today. It is possible to go beyond the stage of analyzing biopsy specimens on slides to imaging the cervix and illuminating the suspicious areas, and even to eliminating the tissue judged to be at risk during a simple examination [1]. Other easily accessible tissues can be screened in a similarly manner [2]. 
The reason for imaging is to discover the location, extent, and "functional capacity" of disease. Among many uses of imaging is disease discovery, but this is not its best use, for several reasons. One reason is that there always is disease smaller than a particular technique can visualize. In addition, the particular technique may not visualize all lesions, which may result in false negatives; normal or benign structures may be mistaken for disease, which may result in false positives. The preferred use of imaging is to diagnose disease that already is suspected. The location may be known because the biochemical signature may suggest the location; or the location may be unknown, which requires more diagnostic acuity in the search for the primary lesion.

Once the location is known, other methods, along with imaging, can be used to elucidate the disease stage and status, which can aid in the prognosis for the patient's outcome. This is what is meant by the more general term "functional capacity" in the above paragraph. The diagram of the stages of cancer development is enhanced with a diagram of possible imaging methods to be employed at various stages through treatment and recurrence.

The ideal imaging method would not only find the lesion and define its apparent size, but also characterize it. This is a tall order. Defining size means to portray the extent of the part of the lesion that can be visualized with the particular imaging method employed. Is the focus of interest the frank lesion with all of the blood vessel changes, rampant tissue growth, and the like that characterize a tumor, or is about the focus all of the tissue that carries the markers of this tumor? Tumor staging today depends in part on lesion size, but reliance on the physical dimensions as seen on a scan or a pathological specimen may be a measure of our ignorance of the real determinants of disease progression, which may depend more on the tissue that carries the markers locally, regionally, and more distantly. Imaging would ideally be able to both visualize the frank lesion and the wider area of affected tissue; it would be a more subtle staging tool than it is today. Clearly, staging today is not perfect; in many cancers it is in a state of constant change, both because new tumor characteristics are revealed that seem to matter to life expectancy and because new treatments require more specificity in the description of the tumor.

Imaging also can be helpful in setting treatment and predicting outcome. The aim is to develop a method to tell immediately after treatment (immediately may be defined as the same day or two weeks later), whether the treatment, if continued as per protocol, will prevent the tumor from being a threat to the patient's health or life.

The first objective often is to show that the tumor is sensitive to the therapeutic method, drug or otherwise, under consideration. That sensitivity should be demonstrated before a long course of therapy is instituted. If the therapeutic attack depends on a biochemical pathway or receptor, the presence of that pathway (and perhaps demonstration of the blocking of other pathways) or receptor must be demonstrated. Imaging can do that, with the added benefit of showing what parts of the tumor can be expected to be susceptible, pointing to the possible use of more than one agent. Therapeutic "cocktails" are used today, but we do not know whether they contain the right agents. If the tumor's sensitivities were known, the cocktail could be designed for maximum effect. This can be done without creating a new drug for each patient, which is unrealistically expensive and time-consuming.

Beyond sensitivity, on which therapeutic efficacy depends, is delivery, both for drugs and other methods. The chemo-therapeutic agent must be shown to reach the tumor and in some quantity. Efficacy often is dependent on the concentration of the agent that reaches the tumor and the amount of time the agent is present. Images of various kinds are able to demonstrate delivery. Some imaging techniques are used in concert with the chemo-therapeutic agents to create the therapy: tumorlocalizing, light-sensitizing compounds can be used in conjunction with laser light delivered locally with a catheter positioned using x-ray computed tomography (CT) or angiography. Bubbles containing therapeutic agents first may be imaged and then burst with ultrasound when they reach the tumor circulation to deliver the agent locally. This capability exists today. In the future, expect a greater necessity to demonstrate delivery, accompanied by more sophisticated chemo-therapeutic agents (e.g., inside special coatings) to promote delivery.

Next comes imaging the behavior of the tumor once it is exposed to the therapy. Early changes in tumor metabolism, blood flow, and cell cycling, for example, can be expected. The correct imaging method will be sensitive to these changes and predictive of further efficacy of the drug during subsequent courses or of the final therapeutic outcome of the therapeutic regimen. It cannot be expected that the same imaging methods that diagnose the tumor initially will be most effective in predicting outcomes. Diagnosis and specification of therapies to be employed will depend on specific bio- 
chemistry, and outcome measures will depend as well on measures of such properties as blood flow, apoptosis, necrosis, and tissue-diffusion properties. Tumor shrinkage is not an immediate enough measure to be helpful.

Because treatment cannot always be expected to be successful, laboratory analytic and imaging methods will be employed to detail recurrence. The recurrent tumor tissue may be very different from the initial tumor and, of course, the tissue milieu may have been disrupted and scarred by excisional biopsy, surgery, necrosis, or radiation therapy. New methods of discovery and diagnosis will need to be developed for this setting; these same methods also could form the basis for following cured patients.

Imaging some regions of the body permits small lesions to be detected; it is not always obvious what course these lesions will follow. It is not known at what point in its development a pre-cancerous nodule or polyp becomes cancerous. In the lung and colon, lesions form into space not occupied by solid structures and may regress. The study of the natural history of nodules in place in the body is so new that we do not know yet what to watch and what to remove. It also is not known what preventative measures may be taken to encourage lesions to regress. This is an area for much research. Imagine the smoker or polyp-prone patient of the future being told not only that he/she has a predilection for cancer, but that adhering to some prescribed measures could decrease the chances greatly; the patient could then be followed by imaging at intervals to see the success of the treatment and regression of lesions. We must be particularly careful not to cause harm in these patients; excessive radiation doses, exploratory surgeries, and the like represent hazards to patients. The immediate risk of some interventions is greater than the long-term risk of cancer.

The above description creates the setting in which the future of imaging is discussed. The major and minor imaging modalities of today are examined for the possibilities that their capabilities can be stretched to be employed in the cancer treatment picture of the future.

\section{What the physical principles will allow, currently and absolutely}

To avoid randomly suggesting futuristic predictions, the major imaging modalities are examined systematically and their possibilities highlighted. Just as the modalities have advanced to varying degrees toward ultimate widespread clinical use, the predictions aim at various points in the life cycle of a technique and detection method. Some modalities now are not much more than "gleams in a few investigators' eyes," and others are represented by thousands of installed units that bear the labels of various major instrument manufacturers. For reasons to be examined, however, modalities also are variously advanced in their exploitation of the techniques of molecular imaging, which means the use of molecular or cellular biological methods in imaging (the term "molecular imaging" has become a form of shorthand that may not yet be understood by all who hear it). Putting thousands of installed units together with new detection techniques that utilize the discoveries of molecular and cellular biology will create a very powerful combination in the future. When prognostications are made, the inherent physical limitations of the detection methods must be kept in mind. For many of these techniques, we look not only at the multidimensional question of the resolution of the technique for the time employed for the measurement (faster measurements often mean poorer resolution), but also consider the contrast of the situation or how well the signal can be measured compared to the background or noise.

The immediately preceding discussion of molecular imaging notwithstanding, I first consider anatomical imaging techniques, which give details about all the tissue in the field of view but do not furnish much information about functional or biochemical processes. $\mathrm{X}$-ray imaging is the "old standard" anatomical imaging technique and is based on the film portrayal of a projection of $\mathrm{x}$-ray attenuation by the tissue illuminated by the X-ray beam. X-ray CT is the more modern modality and shows $\mathrm{X}$-ray attenuation in greater contrast on transaxial images that have been reconstructed by a computer from a collection of projection images detected around the body. As ultrasound and magnetic resonance imaging (MRI) are used in everyday practice, they are both anatomic techniques, each based on their own set of physical principles and dependent on these for the strength and contrast of their signal.

CT is a modality that yields mostly anatomic results; by definition it is able to detect very early disease. However, this modality has captured the public trust and imagination; it is being used by the worried public to look for clinically undetectable disease. This application is not encouraged by the medical profession or insurers because there is no evidence that it improves the outcome for patients or society. CT also is being used to detect early lung and colon cancers in a non-invasive 
way. The population of long-term smokers is at risk for lung cancer; a trial of CT scanning of the lung is underway to test whether CT can detect small lesions in early stages of lung cancer reliably and whether it will save lives [3]. This technique is called "virtual bronchoscopy" by some. Growth of lesions on CT images made several months apart is considered as positive evidence of disease. There are, of course, lung diseases other than cancer that can cause false positives. The same technique used for colon cancer, called "virtual colonoscopy," also now is in trial. It is more invasive in that it requires that the patient first drink purgatives to cleanse the bowel. In both of these CT "-oscopy" techniques, the computer is used not only for the initial reconstruction of the images, but also to present the images in a way that the reader can understand and, further, for an initial interpretation of the images, called computer-assisted diagnosis (CAD). The speed of modern computers has greatly aided in the success of both of these techniques. In the future, more improvements in CAD, as well as more experience with the natural history of the diseases as seen in the CT images, will make it possible for CT to be of great utility in disease detection. Smaller and smaller lesions will be visible as more detectors are added to the instrument; the new scanners have voxels of the same size in all three dimensions, and the overall width of the detectors together maks it possible to image the whole chest in one breath-hold during a helical scan. Three-dimensional (3D) isotropic imaging not only makes possible better 3D presentation, but also better CAD.

CT imaging is further used to aid the bronchoscopist in his/her attempt to biopsy lesions seen on the initial lung images [4] and perhaps to aid in their ablation. Besides biopsy, it also is possible to look at the lesions with a combination of particular wavelengths and internally administered fluorescent compounds that increase the contrast of the malignant lesion. Ablation may be performed by any of a number of methods, but all need to be guided by close-to-real-time imaging to achieve success.

In the case of CT, there not only is the contrast inherent in differences in x-ray attenuation as a function of the energy of the beam, but also the possibility of augmenting the contrast with contrast agents injected into the arteriovenous system or the cerebrospinal fluid space, ingested, instilled into various body cavities, or inhaled. The agents typically are based on high atomic weight elements, because the electrons surrounding the nucleus cause $\mathrm{x}$-ray attenuation. Iodine is a favorite element, but transition metals also are possible. The hazard is that high atomic weight materials often are poisonous and must be kept firmly attached to the particular substance to which they are chemically bonded when they are injected. Another problem to be solved is that image contrast is proportional to the amount of contrast agent present; it takes on the order of micromolar quantities to attenuate the x-rays enough to affect the images. The contrast agents must therefore be non-toxic themselves. There has been very little work in the discovery of new x-ray contrast agents since the advent of the low and non-ionic iodine-based agents of 20 years ago. The challenge, to which a few chemists are rising, is to develop agents of sufficient contrast, low toxicity, and targeted behavior $[5,6]$. Since the gadolinium ion contained in many magnetic resonance (MR) contrast agents is of high enough atomic weight to act as a CT contrast agent, it is possible that, in some situations in which the MR agent is sufficiently concentrated, CT also could be used to image the agent. The advantage of CT is that there is a huge installed base of instruments that can be employed for imaging; modern instruments are fast enough to image the chest in a single breath-hold.

To the extent that anatomic ultrasound and MRI are used in a similar fashion to CT, the general statements made above also apply. All three techniques are much in demand for biopsy guidance today. Most organs are not biopsied blindly, and it can be expected that this will not occur in the prostate for much longer, either. The greatest improvement that might be offered today is to have better control of the biopsy location so that cells taken along the needle track can be used to help create a picture of the anatomic site. We expect in the case of ultrasound that there will be a greater use of contrast agents in the future and that these may be used in novel ways. Ultrasound has the capability of loosing the contents of a bubble at an intended site; this could be a way of dosing for imaging by another modality, for example, as well as a way of inserting a substance that could combine with tumor markers for detection in a subsequent blood or urine sample or an MR or radioactive contrast agent.

Women with a high risk of breast cancer can be imaged with MRI. It provides images via a totally different physical principal than $\mathrm{x}$-ray mammographic images, so the techniques are complementary. Ultrasound may be used in this setting as well. There are clinical trials underway to look into the accuracy of ultrasound and MRI in the setting of women at high risk for breast cancer. Ultrasound and MRI may become part of routine care for these patients, especially because 
these techniques do not deposit the radiation dose of $\mathrm{x}$ ray mammography. As body fluid markers of the most prevalent cancers become more commonplace and as genetic screening to discover true disease probabilities becomes a reality, imaging by CT, MRI, and ultrasound to locate the disease site and aid in biopsy will become even more common.

MR has more potential, than it has exhibited thus far. Manufacturers have been increasing the field strength, although lower field-strength instruments still are in common use. A higher field strength increases the contrast caused by the proton concentration differences, which is the basis for MRI. Increasing the contrast allows faster imaging for the same signal-to-noise ratio. Techniques to make the examination faster, in the form of new sequences or exploitation of yet another property of the protons in the biological setting, have been appearing every year. For example, it is possible to measure the diffusion of water in a field, with tumor tissue displaying different diffusion properties than does normal tissue. We can expect continued improvements in MR imaging. To the extent that each of these improvements is made to a particular manufacturer's instrument, the methods will have to be generalized to all instruments before the improvements can benefit everyone. More compounds will be discovered to be important in the early appearance of cancer and the difference that they represent in the proton spectra of the organ in question will make imaging the organ with magnetic resonance spectral imaging (MRSI) more prevalent; higher field strengths separate the various peaks in the spectra more, again causing better imaging in terms of higher resolution or faster examinations. The ability to reset the MR instrument to image nuclei other than protons allows for the imaging of carbon-13, nitrogen15, phosphorus-33, and fluorine-19. These nuclei can be incorporated in contrast agents for imaging. The process of resetting the MR instrument to image other nuclei has been time consuming. To the extent that imaging other nuclei is perceived to be important, the time to reset the instrument will be reduced until it is no more difficult than dialing the change on a knob.

The nuclear medicine techniques rely on the concentration of minute amounts of imaging agents labeled with radioactivity for their contrast. The possibilities are as great as the capabilities of the chemist to synthesize the compounds and tag them with the radionuclides, because the concentrations needed are so small. The question of the numbers of available compounds hinges not only on the imagination of the radiochemists and their numbers and resources, but also on the imag- ination of the therapeutic drug developers and the research results of those discovering the biological targets, receptors, and pathways involved in disease. Radiochemists cannot carry the whole load but must observe the research results of the molecular and cellular biologists participating with them, but not leading them, in their efforts. Look for many and diverse developments in imaging compounds. Since the best signal registers as a radioactive spot in a non-radioactive background, often referred to as a "hot spot in a cold field," compounds that localize because of unique tumor properties are better than are those that localize in a normal organ and expose a tumor as a non-radioactive or cold area. Positron emission tomography (PET), single photon emission computed tomography (SPECT), and other nuclear medicine techniques also are limited by the resolution and sensitivity of the instruments and the need to use the smallest radiation dose consonant with the possibility of a diagnostic result.

A recurring theme in the design of imaging agents is the creation of a backbone for carrying an imaging agent, to be attached to different moieties to cause the active imaging agent to be localized in different ways. The imaging agents could vary; if the system really works, the mechanism of localization and the concentration would be the same without regard to the imaging agent attached. In some cases, part of the compound can be localized at one time and another part added later. The biotin-streptavidin system works in this fashion, with the localization of the non-radioactive part and clearance of all the material that is not localized over whatever time period this takes. Then the radioactive part is added and couples to the localized part. The radioactivity that is not bound clears quickly, and imaging with high target-to-background ratios can be achieved.

Much of the medically useful spectrum of radioactive nuclides is already in use, so there will not be a great increase in the numbers of nuclides. Were medical cyclotrons to become much cheaper and easier to operate, the very short-lived nuclides, such as oxygen15 , nitrogen-13, and carbon-11, could be put to more widespread use. Fluorine-18 (F-18), with its almost two-hour half-life and low positron energy, is almost ideal for many positron-imaging situations. The compounds that use the F-18 label must localize during two to four hours post injection so that their optimum imaging time occurs within two half-lives. Deposition in the site of interest and the clearing of background material are issues still to be resolved.

Improvements to instruments to image radioactive materials will come from improvements to the detec- 
tors and collimators, computers that are integrated with the instrument, and image-processing and analysis software. PET has an inherent fuzziness that is caused by the energy of the emitted positron; higher energy means that the positron travels farther from the point of its emission before interacting with an electron to annihilate. It is the annihilation radiation that is measured. Computer algorithms will solve this conundrum [7]. For the time being, we are content to observe that there is an inherent resolution proportional caused by the emission energy and spectron; the positron emitters with low energies are chosen for examinations where better resolution is required. The complaint about SPECT is that it is not quantitative. Of course, the complaint is not that there is not some sort of proportionality between the number of counts observed and the amount of activity present, but that it is not a linear relationship because of attenuation, scattering, and partial volume effects. PET, because of years of research applications in which the input and output functions were measured, is now quantitated according to Standard Uptake Value (SUV) methods that have more or less accuracy. Since the SUV depends on a model for the uptake (and excretion) of the material, a model must be developed for each radiopharmaceutical that is used in this way.

Optical methods are developing as research and clinical tools. In everyday use are light and slit lamps to view the skin, eyes, ears, nose, mouth, throat, and vagina to the cervix. Endoscopes have been developed to go farther into the gastrointestinal tract from either end, into the respiratory tract, into the urinary bladder, farther into the female reproductive tract, into the milk ducts of the breast, as well as into the peritoneal cavity through slits in the abdominal wall. These enable many surfaces to be examined with many kinds of light and with much magnification if desired. The data can be viewed in real time or recorded; it may be associated with the site so that the magnification is of a known site, or it may be associated with a biopsy device for sampling the area in question. Contrast agents, applied topically or systemically, can be used to enhance the features of interest. Tissue penetration has been a problem-more or less so depending on the wavelength of the light in use. The lack of penetration caused by scattering and attenuation is a difficult problem for computers to solve. In the future, the use of better lasers of many wavelengths, combined with more sophisticated computer algorithms, will make optical imaging of many body parts commonplace [2]. Contrast agents localized in lung tumor tissue will make it possible to aim biopsy needles from the surface. Prostate cancers will be caused to emit light or fluoresce and make radiation treatment planning or surgery easy. Currently, lymph nodes can be contrasted with methylene blue and seen at the time of surgery; in the future, this will be possible optically without surgical exposure, and choices for nodal biopsy and excision will be made prior to surgery.

Chemically altering optical contrast agents will improve their sensitivity. Altering the structure of green fluorescent protein (GFP) can improve its sensitivity by three times [8], greatly enhancing its utility. GFP requires photoactivation for greatest light fluorescence, but it has a native brightness before activation that can be considered background noise if the objective is to activate only a specific field. Many more altered proteins will be tailored to the circumstances of their use.

To the extent that the use of specially prepared viruses to infect tissues of interest is broadened, it will be possible for these viruses to import the imaging compounds along with the rest of their "payload." These nascent imaging agents could be optical agents that are activated by a specific wavelength of light or receptors for imaging agents of all kinds.

A number of detection methods depend on radiation from other parts of the spectrum. These include near infrared (NIR) imaging [9], magnetic resonance elastography $[10,11]$, optical computed tomography [1215], electrical impedance spectroscopy [16], and microwave imaging $(0.5-3 \mathrm{GHz})$ [16].

Note that, in cancer research, whereas the mouse has been the animal model of choice because of the great possibilities of modeling human cancer, the breast has been the cancer site of choice for testing many techniques. The reasons not only include that breast cancer is a relatively common, diverse, and lethal disease, but also that the breast is so well positioned to allow imaging, biopsy, and observations of the effects of therapy. The "fly in the ointment," of great comfort to patients but not so useful to researchers, is that breast cancer has a fairly long course in many cases; observations must be made over the course of years, and the final outcome is not known for some time. Imaging can serve as a surrogate for the eventual outcome, but only after much research. Thus, those who are looking for the application of new ideas in the screening, diagnosis, and monitoring of therapy should follow the literature of research in breast cancer.

For both human and animal studies that involve ionizing radiation, radiation dose is important. It is desirable to keep the dose as low as possible to avoid extra 
radiation. Some human $\mathrm{x}$-ray studies take enough time that a patient may receive skin burns and other damage; other x-ray studies merely confer a larger dose of radiation than is desirable. $\mathrm{X}$-ray and nuclear medicine studies are the problem areas. Recently, CT scanner manufacturers have become concerned and are creating techniques to minimize radiation dose. In areas of the body with non-uniform attenuation, such as the chest, it is possible to set a desired constant noise level and vary the dose to reach this noise level. Of course, dose can be varied by patient size and by the part of the body being imaged.

Radiation dose is important in animal imaging as well. Of course, there is no governmental authority regulating the use of radiation in animals. Instead, we must use common sense. If an animal is to be used in a continuing experiment, it is only sensible not to give it a radiation dose at each imaging session that is lethal or is the equivalent of a radiation therapy treatment, because the radiation dose would then perturb the experimental results. These ideas must be considered in animal CT, PET, and SPECT. Because count rates, instrument sensitivity and resolution, and the time that it takes to image an animal all are interrelated, the design of the instrument and of the experiment must be considered at the same time. It is easier to change the experiment than the instrument, but an instrument designed for living animals to be imaged multiple times over the course of weeks or months would be a big step toward solving the radiation dose problem.

It is not the purpose of this chapter or this volume to consider the question of diagnostic technology improvement costs and benefits to the health care economy. There are many (I would call them Luddites) who believe that the cost of modern medicine has risen so rapidly because of the cost of the equipment to do elaborate tests, as equipment costs are passed on to the health care system through billings to patients and payers. The opposite side, which I take, is that modern diagnosis has made possible not treating patients who cannot benefit by making possible the division of patients into groups who will benefit and who will not and by making possible earlier diagnoses. Thus, patients' lives improve, allowing them to continue to contribute to the overall economy. To the extent that most medical care is delivered at the end of life, I along with others would decry the waste in this system, but to the extent that we cannot predict who will die and who will survive, some of this expense is necessary.

One of the clear costs of any diagnostic procedure is the cost of the next test or procedure for those with uncertain or positive results. False positives haunt the health care economy and false negatives haunt patients, so we live in a world where a balance is struck between the two.

\section{Where imaging in early detection could go}

The suggestion that the various modalities will be more powerful together rather than separately is not new. Images made by detectors operating according to very different physical principles can supply complementary information. Images that give information only about a specific site of uptake are complemented greatly by images that show the anatomical location of that site, for example. For the future, increasing the possibility of operating the two detectors simultaneously and of fusing the images in space will be a challenge and will provide not only the information we expect but also serendipity.

A very recent example that has not come close to clinical application is the use of diffuse optical tomography in the setting of functional magnetic resonance imaging (fMRI) [17]. Currently, fMRI is used to validate diffuse optical tomographic imaging so that the latter could be used in space during manned space flights to record regional differences and changes in brain blood flow and oxygenation. These same differences are of interest in the study of tumors. There also is the real possibility of using a contrast agent in this setting to enhance both the optical imaging and the synergy between the two very different modalities, as Jeffrey Sutton at the National Space Biomedical Research Institute has done [18]. The publications of the Chance group [19] show the prospects for diffuse optical tomography combined with MRI, including $\mathrm{Gd}^{+3}$ contrast agents, in breast cancer patients.

Instruments that combine PET with CT have achieved commercial status for human use; it has proved more challenging to do this for small animals, but the technique is being perfected [20]. It will be more difficult to combine PET with MRI, but it is being attempted for small-animal imaging [21]. In both cases, the anatomic capabilities of CT and MRI are being exploited to localize the PET activity foci. However, as experience is gained with the combinations, synergy will be found. There are new imaging agents in development for all three modalities. At first the combinations will be used to validate each other, but soon the research will branch out so that perhaps an agent for imaging angiogenesis utilizing one modality 


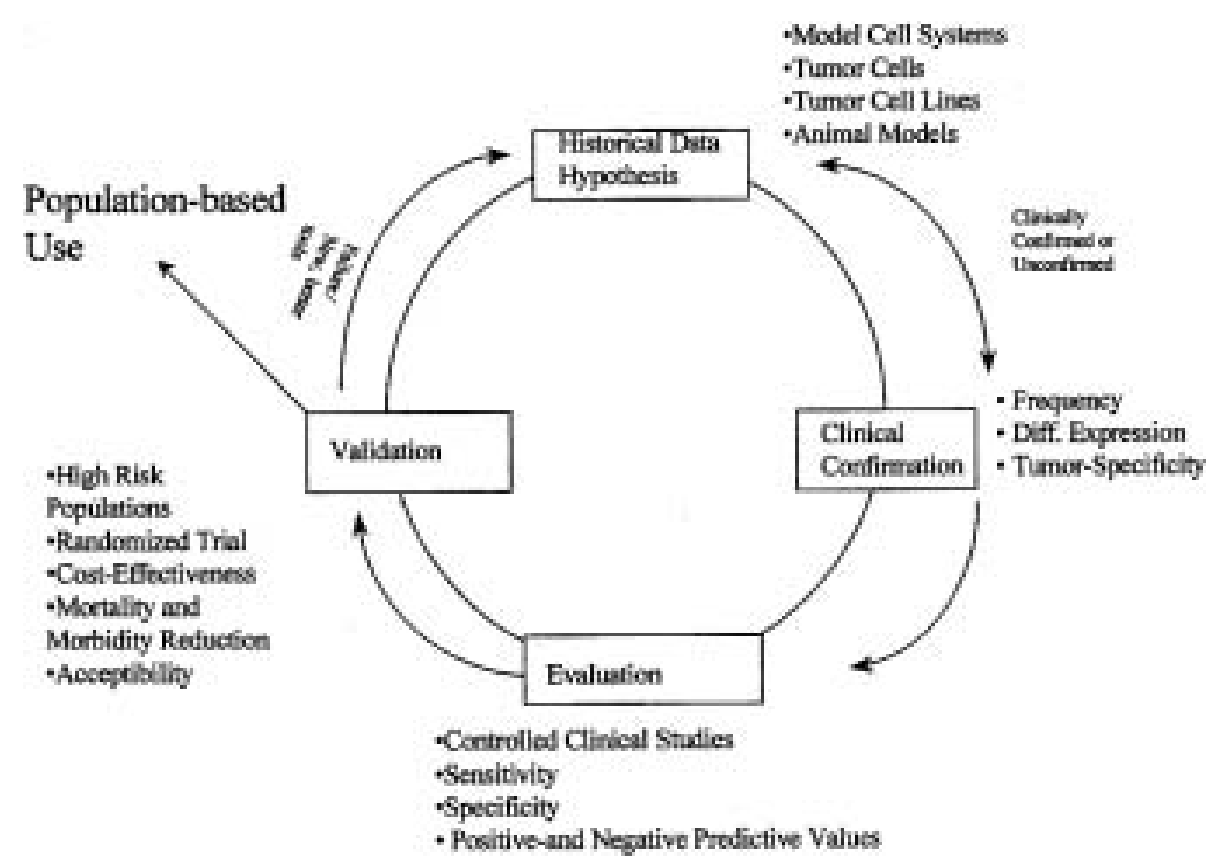

Fig. 2. Developmental cycle for early detection markers and technologies (used with permission [35]).

will be combined with an agent and another modality to image proliferation or a specific tumor marker [22].

Clinical ultrasound in human beings yields fairly fuzzy images of the reflection of sonic waves from tissue barriers and changes; they are the result of hand scanning the subject. The images are hard to correlate with other images in which the detected signal is lined up anatomically. Three-dimensional tomographic ultrasound through tissue changes this and will make it possible to fuse ultrasound images with those from other modalities. There will be positioning issues to be worked out, but the result will be synergy. As mentioned earlier, one of ultrasound's possibilities is to use its power to burst bubbles containing drugs in the locality of interest $[23,24]$.

MRI, in its many versions, creates the possibility that these can be used in concert just as different modalities can be employed together. This is explored in a paper by a number of leading investigators in this area [25].

Another recent article about innovations produced by the National Aeronautics and Space Administration (NASA) details the possibility that the application of an electromagnetic field could target microcapsules injected into the artery perfusing a tumor [26]. The microcapsules would have an outer membrane that is transparent to the specific radiation to be applied and is insoluble in the body. Inside the capsule would be the drug of choice as well as a separate compartment con- taining ferromagnetic particles chosen to be sensitive to heating in the electromagnetic field. The idea would be to inject the microcapsules into an artery that feeds the tumor or organ of choice and turn on the electromagnetic radiation to heat and melt the particles, thereby releasing the drug. Once the possibilities for controlled drug release are known, those same possibilities can be employed for controlled imaging-agent release. In the case of imaging agents, the ferromagnetic particles used to heat and melt the capsules also might be used to image with MRI. Is it too futuristic to imagine encapsulating some of the materials that do not localize well or that metabolize all over the body so that their specific action can be delivered locally? What are the prospects for making an organ fluoresce after such a "treatment" to make its structures shine and the differences from normal come into relief?

Discussions of "smart" contrast agents have become the vogue [27-29]. The meaning of "smart" is that the agent is tuned to change in some way in the presence of the tissue or substance of interest; the change is detected as a change in signal. The smart agent may detect a change in oxygenation, a change in $\mathrm{pH}$, the presence of a particular enzyme [30], calcium content [31], or something else. Li and colleagues refer to the idea of calcium-dependent measurements with MR and the luminescence of aequorin. To date, the typical agent has been detected by optical or MR methods, but more 
familiarity with the idea will promote wider application. The two forms of hemoglobin are the basis for fMRI and for some optical techniques, but research in the area of smart agents is just beginning. For example, there has been little use in radioactive imaging of compounds that shed their activity in certain binding sites and not in others, but this is just as possible as the methods employed by other smart agents.

In some ways, this idea is not dissimilar to the idea of pre-conditioning a site for the delivery of a radioactive substance, such as the use of the avidin-biotin system. The idea of pre-conditioning has not been employed over the spectrum of detection methods. One reason is the differences in sensitivity of the imaging methods, but ways to improve the signal are being developed. In an animal model, Weissleder and his group [32] increased the iron-binding sites on tumors and used super-paramagnetic iron oxide particles bound to the iron-binding sites for an enhanced imaging effect with MRI. It also has been demonstrated that some tumors express an excess of iron-binding sites [30], so there is a real possibility of using this system clinically. Connecting the expression of excess iron-binding sites to a patient's prognosis and tumor stage and perfecting the system for clinical imaging are possible in the future. With these few examples for inspiration, it is possible to imagine many future applications.

The chapter on animal models contains suggestions about what might be expected from animal models in the future. The field will branch out from models that produce predictable disease on a schedule to animals that are less predictable but better exemplify the wide range of human disease. Figure 2 is a schematic of the developmental cycle for early detection markers and technologies. What we have considered in this volume is spread around the developmental circle. The imagers are not the theorists in the area of early detection and the use of markers, but they follow the many leads and exploit them to aid in the discovery process.

\section{References}

[1] K. Sokolov, M. Follen, J. Aaron, I. Pavlova, A. Malpica, R. Lotan and R. Richards-Kortum, Real-time vital optical imaging of precancer using anti-epidermal growth factor receptor antibodies conjugated to gold nanoparticles, Cancer Res 1;63(9) (May, 2003), 1999-2004.

[2] K. Sokolov, M. Follen and R. Richards-Kortum, Optical spectroscopy for detection of neoplasia, Curr Opin Chem Biol 6(5) (Oct. 2002), 651-658. Review.

[3] B.J. Hillman, ACRIN. Economic, legal, and ethical rationales for the ACRIN national lung screening trial of CT screening for lung cancer, Acad Radiol 10(3) (Mar. 2003), 349-350.
[4] A.P. Kiraly, W.E. Higgins, G. McLennan, E.A. Hoffman and J.M. Reinhardt, Three-dimensional human airway segmentation methods for clinical virtual bronchoscopy, Acad Radiol 9(10) (2002), 1153-1168.

[5] B.F. Mullan, M.T. Madsen, L. Messerle, V. Kolesnichenko and J. Kruger, X-ray attenuation coefficients of high-atomicnumber, hexanuclear transition metal cluster compounds: a new paradigm for radiographic contrast agents, Acad Radiol 7(4) (Apr. 2000), 254-259.

[6] J.K. Doerr-Stevens, D.A. Bakan, F.T. Lee Jr, S.G. Chosy, B.K. Markhardt, A.K. Bonneville, C. Burrascano, C. Delaney, M.A. Longino, Y. Greener and J.P. Weichert, Imaging efficacy of a hepatocyte-selective polyiodinated triglyceride (DHOGLE) for contrast-enhanced CT, Acad Radiol 9(1) (May, 2002), S200-S2004.

[7] A. Ruangma, F. Laforest, J.S. Lewis, X. Sun, Y.-C. Tai and M.J. Welch, Initial evaluation of USC-MAP for imaging of radiopharmaceuticals labeled with three copper radionuclides, Mol Imag Biol 5(3) (May/June 2003), 125. Abstract.

[8] J. Lippincott-Schwartz and G.H. Patterson, Development and use of fluorescent protein markers in living cells, Science 300(5616) (Apr. 2003), 87-91.

[9] S. Srinivasan, B.W. Pogue, S. Jiang, H. Dehghani, C. Kogel, S. Soho, J.J. Gibson, T.D. Tosteson, S.P. Poplack and K.D. Paulsen, Interpreting hemoglobin and water concentration, oxygen saturation, and scattering measured in vivo by nearinfrared breast tomography, Proc Natl Acad Sci USA 100(21) (14, Oct. 2003), 12349-12354.

[10] E.E. Van Houten, M.M. Doyley, F.E. Kennedy, J.B. Weaver and K.D. Paulsen, Initial in vivo experience with steady-state subzone-based MR elastography of the human breast, J Magn Reson Imaging 17(1) (Jan. 2003), 72-85.

[11] A.L. McKnight, J.L. Kugel, P.J. Rossman, A. Manduca, L.C. Hartmann and R.L. Ehman, MR elastography of breast cancer: preliminary results, AJR Am J Roentgenol 178(6) (June, 2002), 1411-1417.

[12] Sevick-Muraca-these Disease Markers special issues.

[13] H. Dehghani, B.W. Pogue, S.P. Poplack and K.D. Paulsen, Multiwavelength three-dimensional near-infrared tomography of the breast: initial simulation, phantom, and clinical results, Appl Opt 42(1) (Jan. 2003), 135-145.

[14] V. Ntziachristos, C. Bremer and R. Weissleder, Fluorescence imaging with near-infrared light: new technological advances that enable in vivo molecular imaging, Eur Radiol 13(1) (Jan. 2003), 195-208. Epub 2002 Jul 19. Review.

[15] E.M. Sevick-Muraca, J.P. Houston and M. Gurfinkel, Fluorescence-enhanced, near infrared diagnostic imaging with contrast agents, Curr Opin Chem Biol 6(5) (Oct. 2002), 642650. Review.

[16] D. Li, P.M. Meaney, T.D. Tosteson, S. Jiang, T.E. Kerner, T.O. McBride, B.W. Pogue, A. Hartov and K.D. Paulsen, Comparisons of three alternative breast modalities in a common phantom imaging experiment, Med Phys 30(8) (Aug. 2003), 2194-2205.

[17] C.J. Aine, A conceptual overview and critique of functional neuroimaging techniques in humans: I. MRI/fMRI and PET, Crit Rev in Neurobiol 9(2\&3) (1995), 229-309.

[18] J.P. Labbe, Diffuse optical tomography may enable brain imaging in space, Biophotonics Internat (Sept. 2002), 31-32

[19] V. Ntziachristos, A.G. Yodh, M.D. Schnall and B. Chance, MRI-guided diffuse optical spectroscopy of malignant and benign breast lesions, Neoplasia 4(4) (Jul-Aug, 2002), 347354. 
[20] Cherry, R. Simon, NIH grant 2R01EB000230-04. A micro $\mathrm{CT} / \mathrm{PET}$ scanner for in vivo screening of mice. See the CRISP database for an abstract: http://crisp.cit.nih.gov/.

[21] Croft-these Disease Markers special issues.

[22] D.M. McDonald and P.L. Choyke, Imaging of angiogenesis: from microscope to clinic, Nat Med 9(6) (Jun. 2003), 713-725.

[23] K. Tachibana and S. Tachibana, The use of ultrasound for drug delivery, Echocardiography 18(4) (May, 2001), 323-328. Review.

[24] N. Miyoshi, J.Z. Sostaric and P. Riesz, Correlation between sonochemistry of surfactant solutions and human leukemia cell killing by ultrasound and porphyrins, Free Radic Biol Med 34(6) (Mar. 2003), 710-719.

[25] Z.M. Bhujwalla, D. Artemov, P. Ballesteros, S. Cerdan, R.J. Gillies and M. Solaiyappan, Combined vascular and extracellular $\mathrm{pH}$ imaging of solid tumors, NMR Biomed 15(2) (Apr. 2002), 114-119.

[26] D.R. Morrison and B. Mosier, Lyndon B Johnson Space Center, Houston Texas. Externally triggered microcapsules release drugs in situ. NASA Tech Briefs, April 2002. General URL: http://www.nasatech.com and more specific URL: http://www.nasatech.com/tsp under Bio-Medical category. Abstract URL: http://www.nasatech.com/Briefs/Apr02/ MSC22939.html.

[27] A.Y. Louie, M.M. Huber, E.T. Ahrens, U. Rothbacher, R. Moats, R.E. Jacobs, S.E. Fraser and T.J. Meade, In vivo visualization of gene expression using magnetic resonance imaging, Nat Biotechnol 18(3) (Mar. 2000), 321-325.
[28] D.J. Bornhop, C.H. Contag, K. Licha and C.J. Murphy, Advance in contrast agents, reporters, and detection, J Biomed Opt 6(2) (Apr. 2001), 106-110.

[29] N. Raghunand, C. Howison, A.D. Sherry, S. Zhang and R.J. Gillies, Renal and systemic $\mathrm{pH}$ imaging by contrast-enhanced MRI, Magn Reson Med 49(2) (Feb. 2003), 249-257.

[30] M. Funovics, R. Weissleder and C.H. Tung, Protease sensors for bioimaging. Anal Bioanal Chem. 2003 Sep 3 [Epub ahead of print] all we have right now; keep watching PubMed for citation of print version.

[31] W.H. Li, G. Parigi, M. Fragai, C. Luchinat and T.J. Meade, Mechanistic studies of a calcium-dependent MRI contrast agent, Inorg Chem 29;41(15) (July, 2002), 4018-4024.

[32] A. Moore, L. Josephson, R.M. Bhorade, J.P. Basilion and R. Weissleder, Human transferrin receptor gene as a marker gene for MR imaging, Radiology 221(1) (Oct. 2001), 244-250.

[33] J.G. Liehr and J.S. Jones, Role of iron in estrogen-induced cancer, Curr Med Chem 8(7) (June, 2001), 8390-849.

[34] C.W. Boone, D.E. Henson and G.J. Kelloff, Pathobiology of incipient neoplasia, in: From Molecular Pathology of Early Cancer, S. Srivastava, D.E. Henson and A. Gazdar, eds. IOS Press, Amsterdam, 1999, pp. 4.

[35] K.M. Fong, S. Srivastava, R. Gopal-Srivastava and B.S. Kramer, Molecular genetic basis for early cancer detection and cancer susceptibility, in: From Molecular Pathology of Early Cancer, S. Srivastava, D.E. Henson and A. Gazdar, eds, IOS Press, Amsterdam, 1999, pp. 23. 


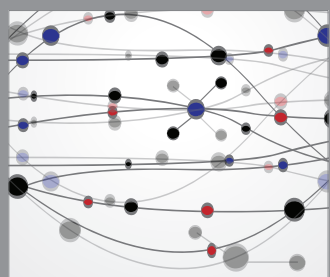

The Scientific World Journal
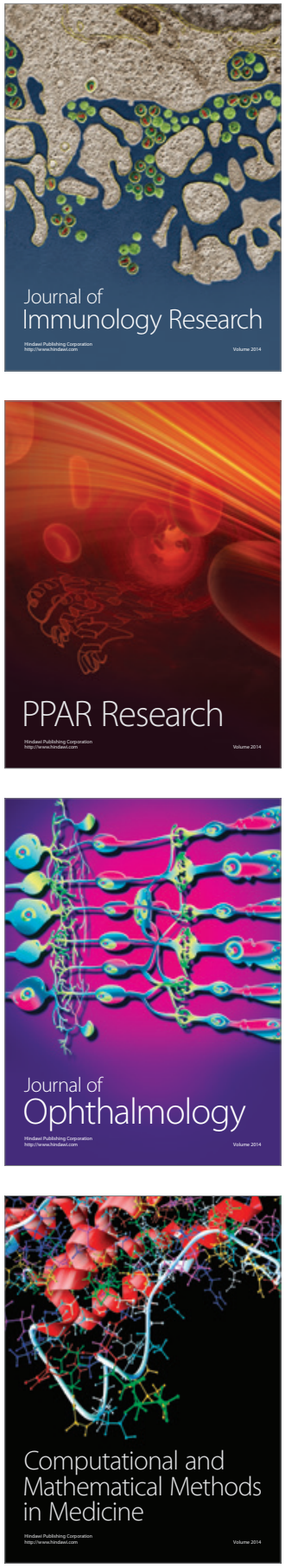

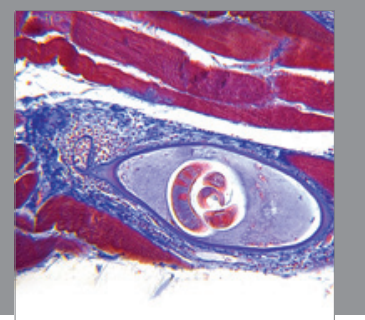

Gastroenterology

Research and Practice
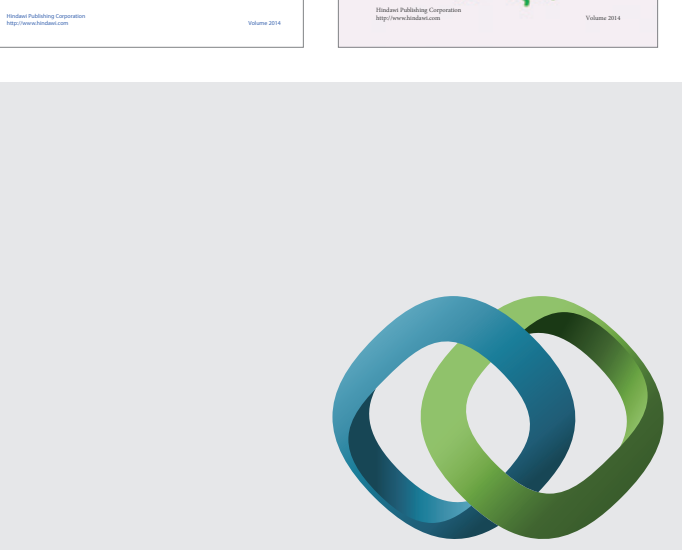

\section{Hindawi}

Submit your manuscripts at

http://www.hindawi.com
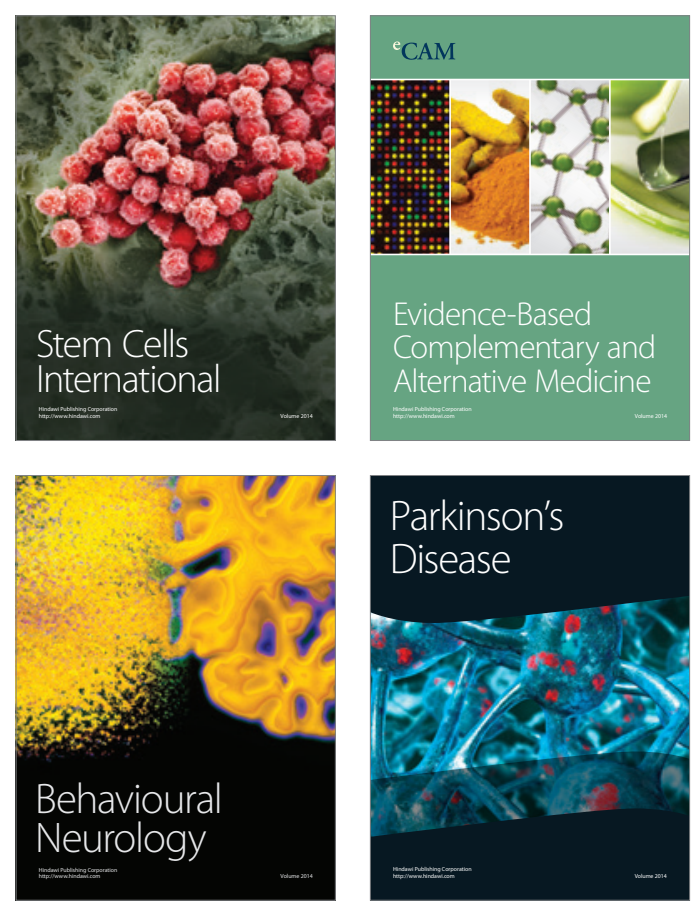

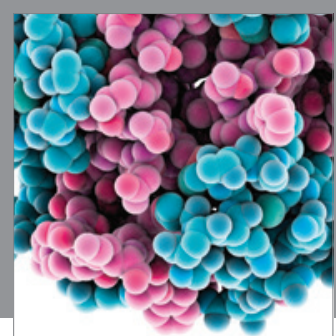

Journal of
Diabetes Research

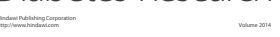

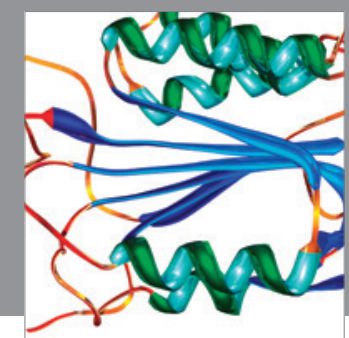

Disease Markers
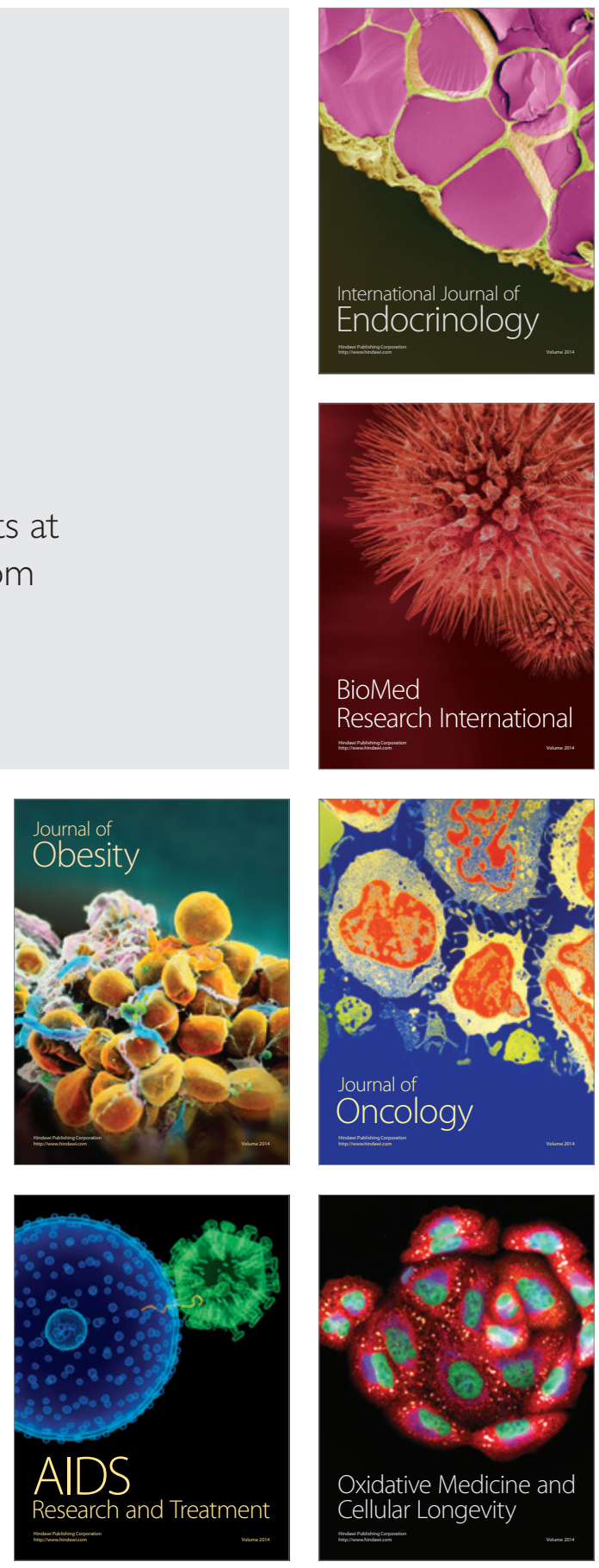